\title{
NUMERICAL NORMALIZATION TECHNIQUES FOR ALL CODIM 2 BIFURCATIONS OF EQUILIBRIA IN ODE'S*
}

\author{
YU. A. KUZNETSOV ${ }^{\dagger}$
}

Abstract. Explicit computational formulas for the coefficients of the normal forms for all codim 2 equilibrium bifurcations of equilibria in autonomous ODEs are derived. They include second-order coefficients for the Bogdanov-Takens bifurcation, third-order coefficients for the cusp and fold-Hopf bifurcations, and coefficients of the fifth-order terms for the generalized Hopf (Bautin) and double Hopf bifurcations. The formulas are independent on the dimension of the phase space and involve only critical eigenvectors of the Jacobian matrix of the right-hand sides and its transpose, as well as multilinear functions from the Taylor expansion of the right-hand sides at the critical equilibrium. The normal form coefficients for the fold-Hopf bifurcation in the "new" Lorenz model are computed using the derived formulas, proving the existence of a nontrivial invariant set in the system.

Key words. normal forms, codim 2 bifurcations

AMS subject classifications. $58 \mathrm{~F} 36,58 \mathrm{~F} 14$

PII. S0036142998335005

1. Introduction. Studying smooth differential equations

$$
\dot{x}=f(x, \alpha), \quad x \in \mathbf{R}^{n}, \quad \alpha \in \mathbf{R}^{m},
$$

usually begins with one-parameter analysis, i.e., constructing the bifurcation diagram of (1.1) with respect to a selected control parameter, say $\alpha_{1}$. In general, there might exist critical parameter values at which the system exhibits codim 1 bifurcations, for example, fold or Hopf bifurcations of equilibrium points. It has been clear since the early 1970s that such analysis is insufficient in many applications because thus obtained one-parameter diagrams revealed strong dependence on other (fixed) parameters $\left(\alpha_{2}, \alpha_{3}, \ldots, \alpha_{m}\right)$. If we allow only one more parameter, say $\alpha_{2}$, to vary, such behavior of one-parameter diagrams can be explained by taking into account so-called codim 2 bifurcation points. These are the points in the $\left(\alpha_{1}, \alpha_{2}\right)$-plane, where several curves corresponding to codim 1 bifurcations intersect transversally or tangentially. A codim 2 point is of particular interest if it is the origin of some equilibrium bifurcation curves and some curves corresponding to bifurcations of periodic orbits (cycles). Such points can be detected by purely local analysis of equilibria and then used to establish the existence of limit cycle bifurcations and other global phenomena that could hardly be proved otherwise.

The theory of codim 2 bifurcations of equilibria in generic systems (1.1) is well developed (see, for example, [1], [6], [10]). A codim 2 bifurcation can be detected along the locus of codim 1 bifurcation points as the change of the dimension of the critical eigenspace (number $n_{c}$ of the eigenvalues of $A=f_{x}$ with $\operatorname{Re} \lambda=0$ ) or as the ranishing of a coefficient of the corresponding normal form of the reduced $n_{c^{-}}$ dimensional system on the center manifold. Then, generically, i.e., under conditions

\footnotetext{
* Received by the editors March 5, 1998; accepted for publication (in revised form) September 25. 1998: published electronically June 3, 1999. This work has been partially supported by the Netherlands Science Foundation (NWO) and has been carried out under the CWI/RIACA project
"Dynamical Systems Laboratory."

hit tp:/www.siam.org/journals/sinum/36-4/33500.html.

vuriacwinl), and Institute of Informatica, Kruislaan 413, 1098 SJ Amsterdam, The Netherlands Pushchino. Moscow Region, 142292 Russia.
} 
that exclude some relationships between the coefficients of the reduced normal form at the codim 2 point and include the transversality to the codim 2 bifurcation manifold in the parameter space, the system (1.1) restricted to the center manifold is smoothly orbitally equivalent to a normal form plus higher order terms. The theory specifies which terms should be kept in the normal form; they are called the resonant terms. In some of the codim 2 cases, truncating higher order terms produces a topologically equivalent system, while in the others, it changes the topology of the bifurcation diagram. In all generic cases, however, the truncated normal forms allow us to predict important features of the system dynamics.

To apply the theory of codim 2 bifurcations to particular models, one needs to verify the nondegeneracy conditions at the bifurcation point, in other words, to compute the critical coefficients of the normal form up to certain order. There are powerful normalization algorithms (for example, [14]) for symbolic computation of the normal form coefficients applicable when the system (1.1) is reduced to the center manifold. However, in most cases the critical parameter values and the equilibrium coordinates are known only approximately, from a numerical analysis, and are not suitable for using any symbolic software. Therefore, numerical normalization techniques have to be developed and implemented into the standard software for the analysis of dynamical systems. For such implementation, one should have as explicit as possible computational formulas for the critical normal form coefficients. These formulas should satisfy the following requirements:

a. They should be independent of the dimension $n$ of the phase space of (1.1).

b. They should involve only critical eigenvalues and eigenvectors of the Jacobian matrix and its transpose.

c. They should be suitable for both numerical and symbolic evaluation.

Notice that there are such explicit formulas for all codim 1 bifurcations of equilibria in generic systems (1.1), i.e., fold and Hopf bifurcations (see section 2).

The aim of this paper is to derive explicit computational formulas for the coefficients of the normal forms for all codim 2 equilibrium bifurcations in (1.1), namely, cusp, Bogdanov-Takens, generalized Hopf (Bautin), fold-Hopf, and double Hopf bifurcations. Some of these coefficients have been obtained earlier by Kurakin and Judovich [9], who gave explicit criteria for stability of equilibria in $n$-dimensional ODEs in some critical cases. Other cubic and fifth-order coefficients of the normal forms for the generalized Hopf, fold-Hopf, and double Hopf bifurcations are irrelevant for the study of stability of the critical equilibrium but determine the topology of the bifurcation diagrams for nearby parameter values. In principle, these coefficients could be found by first computing the Taylor expansion of the center manifold and then evaluating the corresponding normal form coefficients. A numerical method to compute reduced equations on the center manifold is developed in [2]. However, the resulting algorithms are complicated (see, for example, [7] in the generalized Hopf case). Moreover, for the double Hopf case, the fifth-order normal form coefficients seem to be never derived and published even in the four-dimensional case.

In this paper we derive all the coefficients using a reduction or normalization technique by Coullet and Spiegel [3] and MAPLE V.R4 symbolic manipulation software. In this approach, the center-manifold reduction and normalization are performed simultaneously. Surprisingly enough, most formulas are rather compact and allow for straightforward implementation. 
2. Codim 1 normal forms. Suppose (1.1) has an equilibrium $x=0$ at $\alpha=0$ and represent $F(x)=f(x, 0)$ as

$$
\begin{aligned}
F(x)= & A x+\frac{1}{2} B(x, x)+\frac{1}{6} C(x, x, x)+\frac{1}{24} D(x, x, x, x) \\
& +\frac{1}{120} E(x, x, x, x, x)+O\left(\|x\|^{6}\right),
\end{aligned}
$$

where $A=f_{x}(0,0)$ and

$$
\begin{aligned}
B_{i}(x, y) & =\left.\sum_{j, k=1}^{n} \frac{\partial^{2} F_{i}(\xi)}{\partial \xi_{j} \partial \xi_{k}}\right|_{\xi=0} x_{j} y_{k}, \\
C_{i}(x, y, z) & =\left.\sum_{j, k, l=1}^{n} \frac{\partial^{3} F_{i}(\xi)}{\partial \xi_{j} \partial \xi_{k} \partial \xi_{l}}\right|_{\xi=0} x_{j} y_{k} z_{l}, \\
D_{i}(x, y, z, v) & =\left.\sum_{j, k, l, m=1}^{n} \frac{\partial^{4} F_{i}(\xi)}{\partial \xi_{j} \partial \xi_{k} \partial \xi_{l} \partial \xi_{m}}\right|_{\xi=0} x_{j} y_{k} z_{l} v_{m}, \\
E_{i}(x, y, z, v, w) & =\left.\sum_{j, k, l, m, s=1}^{n} \frac{\partial^{5} F_{i}(\xi)}{\partial \xi_{j} \partial \xi_{k} \partial \xi_{l} \partial \xi_{m} \partial \xi_{s}}\right|_{\xi=0} x_{j} y_{k} z_{l} v_{m} w_{s}
\end{aligned}
$$

for $i=1,2, \ldots, n$.

If the equilibrium $x=0$ of (1.1) exhibits a fold bifurcation at $\alpha=0$, the Jacobian matrix $A$ has a simple zero eigenvalue $\lambda_{1}=0$ and no other critical eigenvalues. Introduce two null-vectors:

$$
A q=0, \quad A^{T} p=0,
$$

and normalize them according to

$$
p^{T} q \equiv\langle p, q\rangle=1 .
$$

The restriction of (1.1) at $\alpha=0$ to the one-dimensional center manifold has the form

$$
\dot{w}=a w^{2}+O\left(|w|^{3}\right), \quad w \in \mathbf{R}^{1},
$$

where the coefficient $a$ can be computed by the formula

$$
a=\frac{1}{2}\langle p, B(q, q)\rangle .
$$

If $a \neq 0$ and (1.1) depends generically on the parameter $\alpha_{1}$, its restriction to the (parameter-dependent) center manifold is locally topologically equivalent to the normal form

$$
\dot{w}=\beta_{1}+a w^{2},
$$

where $\beta_{1}$ is the unfolding parameter. This normal form predicts the collision of two equilibria when the parameter $\beta_{1}$ passes zero.

If the equilibrium $x=0$ of (1.1) exhibits a Hopf bifurcation at $\alpha=0$, the Jacobian matrix $A=f_{x}(0,0)$ has a simple pair of purely imaginary eigenvalues $\lambda_{1,2}= \pm i \omega_{0}, \omega_{0}>0$, and no other critical eigenvalues. Introduce two complex vectors:

$$
A q=i \omega_{0} q, \quad A^{T} p=-i \omega_{0} p,
$$


and normalize them according to

$$
\bar{p}^{T} q \equiv\langle p, q\rangle=1
$$

The restriction of (1.1) at $\alpha=0$ to the two-dimensional center manifold is locally smoothly orbitally equivalent to the complex normal form

$$
\dot{w}=i \omega_{0} w+l_{1} w|w|^{2}+O\left(|w|^{4}\right), \quad w \in \mathbf{C}^{1},
$$

where the normal form coefficient $l_{1}$ can be computed by the formula

(2.5) $l_{1}=\frac{1}{2} \operatorname{Re}\left\langle p, C(q, q, \bar{q})+B\left(\bar{q},\left(2 i \omega_{0} I_{n}-A\right)^{-1} B(q, q)\right)-2 B\left(q, A^{-1} B(q, \bar{q})\right)\right\rangle$,

where $I_{n}$ is the unit $n \times n$ matrix. If the first Lyapunov coefficient $l_{1} \neq 0$ and (1.1) depends generically on the parameter $\alpha_{1}$, its restriction to the (parameter-dependent) center manifold is locally topologically equivalent to the normal form

$$
\dot{w}=\left(\beta_{1}+i \omega_{0}\right) w+l_{1} w|w|^{2} .
$$

This normal form describes a bifurcation of the unique limit cycle from the equilibrium $w=0$, when the parameter $\beta_{1}$ passes the bifurcation value $\beta_{1}=0$. The direction of the bifurcation is determined by the sign of $l_{1}$.

The formulas (2.3) and (2.5) are derived using the center manifold reduction and subsequent normalization on the center manifold in [10]. Originally, an expression equivalent to (2.5) was obtained using the Lyapunov-Schmidt reduction and asymptotic expansions for the bifurcating cycle by Kopell and Howard in [13] and by van Gils [16]. These formulas will be rederived below as a part of codim 2 analysis. The formulas (2.3) and (2.5) are implemented in CONTENT [11].

3. List of the codim 2 normal forms. In generic systems (1.1), only five codim 2 bifurcations of equilibria are possible [1], [6], [10]:

(i) $\operatorname{Cusp}\left(\lambda_{1}=0, a=0\right)$. The restricted-to-the-center manifold at the critical parameter values equation reads

$$
\dot{w}=c w^{3}+O\left(w^{4}\right), \quad w \in \mathbf{R}^{1} .
$$

If $c \neq 0$ and the system (1.1) depends generically on two parameters $\left(\alpha_{1}, \alpha_{2}\right)$, its restriction to the center manifold is locally topologically equivalent near the bifurcation to the normal form

$$
\dot{w}=\beta_{1}+\beta_{2} w+c w^{3},
$$

where $\beta_{1}$ and $\beta_{2}$ are the unfolding parameters. This normal form predicts a hysteresis phenomenon near the bifurcation.

(ii) Bogdanov-Takens $\left(\lambda_{1,2}=0\right)$. The restriction of (1.1) to the center manifold at the critical parameter values is locally smoothly equivalent to the normal form

$$
\left\{\begin{array}{l}
\dot{w}_{0}=w_{1}, \\
\dot{w}_{1}=a w_{0}^{2}+b w_{0} w_{1}+O\left(\|w\|^{3}\right),
\end{array}\right.
$$

where $w=\left(w_{0}, w_{1}\right)^{T} \in \mathbf{R}^{2}$. If $a b \neq 0$ and the parameters $\left(\alpha_{1}, \alpha_{2}\right)$ enter (1.1) generically, the restricted system is locally topologically equivalent to the normal form

$$
\left\{\begin{array}{l}
\dot{w}_{0}=w_{1} \\
\dot{w}_{1}=\beta_{1}+\beta_{2} w_{0}+a w_{0}^{2}+b w_{0} w_{1} .
\end{array}\right.
$$


Analysis of this normal form reveals a curve in the parameter plane emanating from the codim 2 point and corresponding to a saddle homoclinic bifurcation: The unique limit cycle born in the Hopf bifurcation approaches the homoclinic orbit and disappears while its period $T \rightarrow \infty$.

(iii) Generalized $\operatorname{Hopf}\left(\lambda_{1,2}= \pm i \omega_{0}, l_{1}=0\right)$. The restriction of (1.1) to the center manifold at the critical parameter values is locally smoothly orbitally equivalent to the normal form

$$
\dot{w}=i \omega_{0} w+l_{2} w|w|^{4}+O\left(|w|^{6}\right), \quad w \in \mathbf{C}^{1},
$$

where the second Lyapunov coefficient $l_{2}$ is real. If $l_{2} \neq 0$ then, generically, the restricted system (1.1) is locally topologically equivalent to the normal form

$$
\dot{w}=\left(\beta_{1}+i \omega_{0}\right) w+\beta_{2} w|w|^{2}+l_{2} w|w|^{4} .
$$

This normal form predicts the existence of a curve originating at the codim 2 point in the parameter plane, where two limit cycles collide and disappear through a nonhyperbolic cycle with a nontrivial multiplier $\mu_{1}=1$.

(iv) Fold-Hopf $\left(\lambda_{1}=0, \lambda_{2,3}= \pm i \omega_{0}\right)$. The normalized restriction of (1.1) to the center manifold at the critical parameter values has the form

$$
\left\{\begin{aligned}
\dot{w}_{0}= & \frac{1}{2} G_{200} w_{0}^{2}+G_{011}\left|w_{1}\right|^{2}+\frac{1}{6} G_{300} w_{0}^{3} \\
& +G_{111} w_{0}\left|w_{1}\right|^{2}+O\left(\left\|\left(w_{0}, w_{1}, \bar{w}_{1}\right)\right\|^{4}\right), \\
\dot{w}_{1}= & i w_{0} w_{1}+G_{110} w_{0} w_{1}+\frac{1}{2} G_{210} w_{0}^{2} w_{1}+\frac{1}{2} G_{021} w_{1}\left|w_{1}\right|^{2} \\
& +O\left(\left\|\left(w_{0}, w_{1}, \bar{w}_{1}\right)\right\|^{4}\right) .
\end{aligned}\right.
$$

Here $w_{0} \in \mathbf{R}^{1}, w_{1} \in \mathbf{C}^{1}$, and the coefficients $G_{k l m}$ in the first equation are real, while those in the second equation are complex. If $G_{200} G_{011} \neq 0$, the restricted-to-thecenter manifold system (1.1) is locally smoothly orbitally equivalent to the system

$$
\left\{\begin{array}{l}
\dot{u}=\beta_{1}+b u^{2}+c|z|^{2}+O\left(\|(u, z, \bar{z})\|^{4}\right), \\
\dot{z}=\left(\beta_{2}+i \omega\right) z+d u z+e u^{2} z+O\left(\|(u, z, \bar{z})\|^{4}\right),
\end{array}\right.
$$

where $\omega, b, c$, and $e$ are real functions of $\beta$, while $d$ is a complex function of $\beta$ :

$$
\omega(0)=\omega_{0}, \quad b(0)=\frac{1}{2} G_{200}, \quad c(0)=G_{011}, \quad d(0)=G_{110}-i \omega_{0} \frac{G_{300}}{3 G_{200}},
$$

and

$$
e(0)=\frac{1}{2} \operatorname{Re}\left[G_{210}+G_{110}\left(\frac{\operatorname{Re} G_{021}}{G_{011}}-\frac{G_{300}}{G_{200}}+\frac{G_{111}}{G_{011}}\right)-\frac{G_{021} G_{200}}{2 G_{011}}\right] .
$$

In general, the $O$-terms cannot be truncated, since they affect the topology of the bifurcation diagram of the system near the bifurcation. Depending upon the coefficients $b, c, d$, and $e$, the system can have two-dimensional invariant tori and chaotic motions and exhibit Neimark-Sacker bifurcations of cycles and Shil'nikov homoclinic bifurcations.

(v) Double Hopf $\left(\lambda_{1,2}= \pm i \omega_{1}, \lambda_{3,4}= \pm i \omega_{2}\right)$. Assume that

$$
k \omega_{1} \neq l \omega_{2}, \quad k, l>0, \quad k+l \leq 5 .
$$


Then, the normalized system (1.1) on the center manifold has the form

$$
\left\{\begin{aligned}
\dot{w}_{1}= & i \omega_{1} w_{1}+\frac{1}{2} G_{2100} w_{1}\left|w_{1}\right|^{2}+G_{1011} w_{1}\left|w_{2}\right|^{2} \\
& +\frac{1}{12} G_{3200} w_{1}\left|w_{1}\right|^{4}+\frac{1}{2} G_{2111} w_{1}\left|w_{1}\right|^{2}\left|w_{2}\right|^{2}+\frac{1}{4} G_{1022} w_{1}\left|w_{2}\right|^{4} \\
& +O\left(\left\|\left(w_{1}, \bar{w}_{1}, w_{2}, \bar{w}_{2}\right)\right\|^{6}\right), \\
\dot{w}_{2}= & i \omega_{2} w_{2}+G_{1110} w_{2}\left|w_{1}\right|^{2}+\frac{1}{2} G_{0021} w_{2}\left|w_{2}\right|^{2} \\
& +\frac{1}{4} G_{2210} w_{2}\left|w_{1}\right|^{4}+\frac{1}{2} G_{1121} w_{2}\left|w_{1}\right|^{2}\left|w_{2}\right|^{2}+\frac{1}{12} G_{0032} w_{2}\left|w_{2}\right|^{4} \\
& +O\left(\left\|\left(w_{1}, \bar{w}_{1}, w_{2}, \bar{w}_{2}\right)\right\|^{6}\right),
\end{aligned}\right.
$$

where $G_{j k l m} \in \mathbf{C}^{1}$. Moreover, if

$$
\left(\operatorname{Re} G_{2100}\right)\left(\operatorname{Re} G_{1011}\right)\left(\operatorname{Re} G_{1110}\right)\left(\operatorname{Re} G_{0021}\right) \neq 0,
$$

the system (1.1) is locally smoothly orbitally equivalent near the bifurcation to the system

$$
\left\{\begin{aligned}
\dot{v}_{1}= & \left(\beta_{1}+i \omega_{1}\right) v_{1}+\frac{1}{2} P_{11} v_{1}\left|v_{1}\right|^{2}+P_{12} v_{1}\left|v_{2}\right|^{2} \\
& +i R_{1} v_{1}\left|v_{1}\right|^{4}+\frac{1}{4} S_{1} v_{1}\left|v_{2}\right|^{4}+O\left(\left\|\left(v_{1}, \bar{v}_{1}, v_{2}, \bar{v}_{2}\right)\right\|^{6}\right) \\
\dot{v}_{2}= & \left(\beta_{2}+i \omega_{2}\right) v_{2}+P_{21} v_{2}\left|v_{1}\right|^{2}+\frac{1}{2} P_{22} v_{2}\left|v_{2}\right|^{2} \\
& +\frac{1}{4} S_{2} v_{2}\left|v_{1}\right|^{4}+i R_{2} v_{2}\left|v_{2}\right|^{4}+O\left(\left\|\left(v_{1}, \bar{v}_{1}, v_{2}, \bar{v}_{2}\right)\right\|^{6}\right)
\end{aligned}\right.
$$

where $\left(v_{1}, v_{2}\right)^{T} \in \mathrm{C}^{2}$ and $P_{j k}$ and $S_{k}$ are complex, while $R_{k}$ are real. Moreover, the real parts of the critical values are given by the expressions:

$\operatorname{Re} P_{11}=\operatorname{Re} G_{2100}, \operatorname{Re} P_{12}=\operatorname{Re} G_{1011}, \quad \operatorname{Re} P_{21}=\operatorname{Re} G_{1110}, \quad \operatorname{Re} P_{22}=\operatorname{Re} G_{0021}$,

and

$\operatorname{Re} S_{1}=\operatorname{Re} G_{1022}+\frac{1}{3} \operatorname{Re} G_{1011}\left[6 \frac{\operatorname{Re} G_{1121}}{\operatorname{Re} G_{1110}}-4 \frac{\operatorname{Re} G_{0032}}{\operatorname{Re} G_{0021}}-\frac{\left(\operatorname{Re} G_{3200}\right)\left(\operatorname{Re} G_{0021}\right)}{\left(\operatorname{Re} G_{2100}\right)\left(\operatorname{Re} G_{1110}\right)}\right]$,

$\operatorname{Re} S_{2}=\operatorname{Re} G_{2210}+\frac{1}{3} \operatorname{Re} G_{1110}\left[6 \frac{\operatorname{Re} G_{2111}}{\operatorname{Re} G_{1011}}-4 \frac{\operatorname{Re} G_{3200}}{\operatorname{Re} G_{2100}}-\frac{\left(\operatorname{Re} G_{2100}\right)\left(\operatorname{Re} G_{0032}\right)}{\left(\operatorname{Re} G_{1011}\right)\left(\operatorname{Re} G_{0021}\right)}\right]$.

As in the fold-Hopf case, the $O$-terms cannot be truncated, since they affect the topology of the bifurcation diagram of the system. Depending on the values of the normal form coefficients, the system can exhibit invariant tori and chaotic motions, as well as Neimark-Sacker bifurcations of cycles and Shil'nikov homoclinic bifurcations.

Proofs of the results formulated above could be found in [10] with all relevant bifurcation diagrams and bibliographical references.

4. The method. The following normalization technique is essentially due to Coullet and Spiegel [3] (see also [4]). Suppose the system (1.1) has, at $\alpha=0$, the equilibrium $x=0$ such that the Jacobian matrix $A=f_{x}(0,0)$ has $n_{c}$ eigenvalues counting multiplicities with zero real part, and denote by $T^{c}$ the corresponding generalized critical eigenspace of $A$. Write the system at $\alpha=0$ as

$$
\dot{x}=F(x), \quad x \in \mathbf{R}^{n},
$$


where $F$ is given by (2.1), and restrict it to its $n_{c}$-dimensional invariant center manifold parametrized by $w \in \mathbf{R}^{n_{c}}$ :

$$
x=H(w), \quad H: \mathbf{R}^{n_{c}} \rightarrow \mathbf{R}^{n} .
$$

The restricted equation can be written as

$$
\dot{w}=G(w), \quad G: \mathbf{R}^{n_{c}} \rightarrow \mathbf{R}^{n_{c}} .
$$

Substitution of (4.2) and (4.3) into (4.1) gives the following homological equation:

$$
H_{w}(w) G(w)=F(H(w)) .
$$

Now expand the functions $G, H$ in (4.4) into multivariant Taylor series,

$$
G(w)=\sum_{|\nu| \geq 1} \frac{1}{\nu !} g_{\nu} w^{\nu}, \quad H(w)=\sum_{|\nu| \geq 1} \frac{1}{\nu !} h_{\nu} w^{\nu},
$$

and assume that the restricted equation (4.3) is put into the normal form up to a certain order. The coefficients $g_{\nu}$ of the normal form (4.3) and the coefficients $h_{\nu}$ of the Taylor expansion for $H(w)$ are unknown but can be found from (4.4) by a recurrent procedure, from lower to higher order terms. ${ }^{1}$ Collecting the coefficients of the $w^{\nu}$-terms in (4.4) gives a linear system for the coefficient $h_{\nu}$,

$$
L h_{\nu}=R_{\nu} .
$$

Here the matrix $L$ is determined by the Jacobian matrix $A$ and its critical eigenvalues, while the right-hand side $R_{\nu}$ depends on the coefficients of $G$ and $H$ of order less than or equal to $|\nu|$, as well on the terms of order less than or equal to $|\nu|$ of the Taylor expansion (2.1) for $F$. When $R_{\nu}$ involves only known quantities, the system (4.5) has a solution because either $L$ is nonsingular or $R_{\nu}$ satisfies Fredholm's solvability condition

$$
\left\langle p, R_{\nu}\right\rangle=0
$$

where $p$ is a null-vector of the adjoint matrix $\bar{L}^{T}$. When $R_{\nu}$ depends on the unknown coefficient $g_{\nu}$ of the normal form, $L$ is singular and the above solvability condition gives the expression for $g_{\nu}$.

For all codim 2 bifurcations except Bogdanov-Takens, the invariant subspace of $L\left(\bar{L}^{T}\right)$ corresponding to the zero eigenvalue is one-dimensional in $\mathbf{C}^{n}$; i.e., there are unique (up to scaling) null-vectors $q$ and $p$,

$$
L q=0, \quad \bar{L}^{T} p=0, \quad\langle p, q\rangle=1,
$$

and no generalized null-vectors. Then, the unique solution $h_{\nu}$ to (4.5) satisfying $\left\langle p, h_{\nu}\right\rangle=0$ can be obtained by solving the following nonsingular $(n+1)$-dimensional bordered system:

\footnotetext{
${ }^{1}$ Obviously, one has $\sum_{|\nu|=1} h_{\nu} w^{\nu} \in T^{c}$.
} 


$$
\left(\begin{array}{cc}
L & q \\
\bar{p}^{T} & 0
\end{array}\right)\left(\begin{array}{c}
h_{\nu} \\
s
\end{array}\right)=\left(\begin{array}{c}
R_{\nu} \\
0
\end{array}\right)
$$

(the idea is due to Keller [8]; see [5] for generalizations). We write $h_{\nu}=L^{I N V} R_{\nu}$.

The Taylor expansion of $H(w)$ simultaneously defines the expansions of the center manifold, the normalizing transformation on it, and the normal form itself. Since we know which terms are present in the normal form a priori, the described procedure is a powerful tool to compute their coefficients at the bifurcation parameter values. In the following sections, this method will be applied to all codim 2 cases.

5. Cusp bifurcation. At this bifurcation, the system (1.1) has an equilibrium with a simple zero eigenvalue $\lambda_{1}=0$ and no other critical eigenvalues. Let $q, p \in \mathbf{R}^{n}$ satisfy

$$
A q=0, \quad A^{T} p=0, \quad\langle p, q\rangle=1 .
$$

Any point $y \in T^{c}$ can be represented as $y=w q, w \in \mathbf{R}^{1}$, where $w=\langle p, y\rangle$. The homological equation (4.4) has the form

$$
H_{w} \dot{w}=F(H(w))
$$

where

$$
\begin{aligned}
& F(H)=A H+\frac{1}{2} B(H, H)+\frac{1}{6} C(H, H, H)+O\left(\|H\|^{4}\right) \\
& H(w)=w q+\frac{1}{2} h_{2} w^{2}+\frac{1}{6} h_{3} w^{3}+O\left(w^{4}\right),
\end{aligned}
$$

$h_{i} \in \mathbf{R}^{n}$, and

$$
\dot{w}=b w^{2}+c w^{3}+O\left(w^{4}\right)
$$

with unknown coefficients $b$ and $c$. Substituting these expressions into the homological equation gives

$$
\begin{aligned}
b w^{2} q+\left(c q+b h_{2}\right) w^{3}= & \frac{1}{2} w^{2}\left[A h_{2}+B(q, q)\right] \\
& +\frac{1}{6} w^{3}\left[A h_{3}+3 B\left(q, h_{2}\right)+C(q, q, q)\right]+O\left(w^{4}\right) .
\end{aligned}
$$

The $w^{2}$-terms in (5.1) give the equation for $h_{2}$ :

$$
A h_{2}=-B(q, q)+2 b q,
$$

where the matrix $A$ is obviously singular. The solvability of this system implies

$$
\langle p,-B(q, q)+2 b q\rangle=-\langle p, B(q, q)\rangle+2 b\langle p, q\rangle=0
$$

and allows one to find $b$, namely,

$$
b=\frac{1}{2}\langle p, B(q, q)\rangle,
$$

as specified by the formula (2.3) for the fold bifurcation. With this value of $b$, the linear system (5.2) becomes

$$
A h_{2}=-B(q, q)+\langle p, B(q, q)\rangle q
$$


and its unique solution $h_{2}=-A^{I N V}[B(q, q)-\langle p, B(q, q)\rangle q]$ satisfying $\left\langle p, h_{2}\right\rangle=0$ can be computed by solving the nonsingular $(n+1)$-dimensional bordered system

$$
\left(\begin{array}{cc}
A & q \\
p^{T} & 0
\end{array}\right)\left(\begin{array}{c}
h_{2} \\
s
\end{array}\right)=\left(\begin{array}{c}
-B(q, q)+\langle p, B(q, q)\rangle q \\
0
\end{array}\right)
$$

Collecting the $w^{3}$-terms in (5.1) yields

$$
c q+b h_{2}=\frac{1}{6} A h_{3}+\frac{1}{2} B\left(q, h_{2}\right)+\frac{1}{6} C(q, q, q),
$$

which is equivalent to another singular system,

$$
A h_{3}=c q+b h_{2}-\frac{1}{6}\left[C(q, q, q)+3 B\left(q, h_{2}\right)\right] .
$$

Its solvability implies

$$
c\langle p, q\rangle+b\left\langle p, h_{2}\right\rangle-\frac{1}{6}\left\langle p, C(q, q, q)+3 B\left(q, h_{2}\right)\right\rangle=0 .
$$

Since $\left\langle p, h_{2}\right\rangle=0$, we obtain the following expression for the coefficient $c$ :

$$
c=\frac{1}{6}\left\langle p, C(q, q, q)+3 B\left(q, h_{2}\right)\right\rangle .
$$

Now recall that $b=0$ at the cusp bifurcation. Under this condition, the coefficient $c$ in the normal form (3.1) can be expressed shortly as

$$
c=\frac{1}{6}\left\langle p, C(q, q, q)-3 B\left(q, A^{I N V} B(q, q)\right)\right\rangle .
$$

6. Bogdanov-Takens bifurcation. At this bifurcation, the system (1.1) has a double zero eigenvalue $\lambda_{1,2}=0$ and there exist two real linearly independent (generalized) eigenvectors, $q_{0,1} \in \mathbf{R}^{n}$, such that

$$
A q_{0}=0, \quad A q_{1}=q_{0} .
$$

Moreover, there exist similar vectors $p_{1,0} \in \mathbf{R}^{n}$ of the transposed matrix $A^{T}$ :

$$
A^{T} p_{1}=0, \quad A^{T} p_{0}=p_{1} .
$$

One can select these vectors to satisfy

$$
\left\langle q_{0}, p_{0}\right\rangle=\left\langle q_{1}, p_{1}\right\rangle=1, \quad\left\langle q_{1}, p_{0}\right\rangle=\left\langle q_{0}, p_{1}\right\rangle=0 .
$$

Any vector $y \in T^{c}$ can be uniquely represented as $y=w_{0} q_{0}+w_{1} q_{1}$, where $w_{0}=\left\langle p_{0}, y\right\rangle, w_{1}=\left\langle p_{1}, y\right\rangle$. The homological equation (4.4) has the form

$$
H_{w_{0}} \dot{w}_{0}+H_{w_{1}} \dot{w}_{1}=F\left(H\left(w_{0}, w_{1}\right)\right)
$$

where

$$
\begin{aligned}
F(H) & =A H+\frac{1}{2} B(H, H)+O\left(\|H\|^{3}\right), \\
H\left(w_{0}, w_{1}\right) & =w_{0} q_{0}+w_{1} q_{1}+\frac{1}{2} h_{20} w_{0}^{2}+h_{11} w_{0} w_{1}+\frac{1}{2} h_{02} w_{1}^{2} \\
& +O\left(\left\|\left(w_{0}, w_{1}\right)\right\|^{3}\right)
\end{aligned}
$$


with $h_{j k} \in \mathbf{R}^{n}$, and $\dot{w}_{0}, \dot{w}_{1}$ are defined by the normal form (3.2) with unknown coefficients $a$ and $b$. Substituting these expressions into (6.1) and collecting the $w_{0}^{2}$ terms, give the singular linear system for $h_{20}$ :

$$
A h_{20}=2 a q_{1}-B\left(q_{0}, q_{0}\right)
$$

The solvability condition for this system is

$$
\left\langle p_{1}, 2 a q_{1}-B\left(q_{0}, q_{0}\right)\right\rangle=2 a\left\langle p_{1}, q_{1}\right\rangle-\left\langle p_{1}, B\left(q_{0}, q_{0}\right)\right\rangle=0
$$

which gives

$$
a=\frac{1}{2}\left\langle p_{1}, B\left(q_{0}, q_{0}\right)\right\rangle
$$

Taking the scalar product of both sides of (6.2) with $p_{0}$ yields $\left\langle p_{0}, A h_{20}\right\rangle=2 a\left\langle p_{0}, q_{1}\right\rangle-$ $\left\langle p_{0}, B\left(q_{0}, q_{0}\right)\right\rangle$, which implies

$$
\left\langle p_{1}, h_{20}\right\rangle=-\left\langle p_{0}, B\left(q_{0}, q_{0}\right)\right\rangle
$$

The $w_{0} w_{1}$-terms in $(6.1)$ give the linear system

$$
A h_{11}=h_{20}+b q_{1}-B\left(q_{0}, q_{1}\right) .
$$

Its solvability means

$$
\left\langle p_{1}, h_{20}+b q_{1}-B\left(q_{0}, q_{1}\right)\right\rangle=\left\langle p_{1}, h_{20}\right\rangle+b\left\langle p_{1}, q_{1}\right\rangle-\left\langle p_{1}, B\left(q_{0}, q_{1}\right)\right\rangle=0 .
$$

Taking into account (6.3), we get

$$
b=\left\langle p_{0}, B\left(q_{0}, q_{0}\right)\right\rangle+\left\langle p_{1}, B\left(q_{0}, q_{1}\right)\right\rangle .
$$

The coefficients $a, b$ of the normal form (3.2) are computed.

7. Bautin (generalized Hopf) bifurcation. At this bifurcation, the system (1.1) has an equilibrium with a simple pair of purely imaginary eigenvalues $\lambda_{1,2}=$ $\pm i \omega_{0}, \omega_{0}>0$, and no other critical eigenvalues. As in the simple Hopf case, introduce two complex eigenvectors:

$$
A q=i \omega_{0} q, \quad A^{T} p=-i \omega_{0} p
$$

and normalize them according to

$$
\langle p, q\rangle=1
$$

Any vector $y \in T^{c} \subset \mathbf{R}^{n}$ can be represented as $y=w q+\bar{w} \bar{q}$, where $w=\langle p, y\rangle \in \mathbf{C}^{1}$. The homological equation (4.4) now takes the form

$$
H_{w} \dot{w}+H_{\bar{w}} \dot{\bar{w}}=F(H(w, \bar{w})),
$$

where $F$ is given by (2.1),

$$
H(w, \bar{w})=w q+\bar{w} \bar{q}+\sum_{2 \leq j+k \leq 5} \frac{1}{j ! k !} h_{j k} w^{j} \bar{w}^{k}+O\left(|w|^{6}\right)
$$


with $h_{j k} \in \mathbf{C}^{n}, h_{k j}=\bar{h}_{j k}$, and

$$
\dot{w}=i \omega_{0} w+\frac{1}{2} G_{21} w|w|^{2}+\frac{1}{12} G_{32} w|w|^{4}+O\left(|w|^{6}\right),
$$

where $G_{j k} \in \mathrm{C}^{1}$.

Collecting the coefficients of the quadratic terms in (7.1) and solving appearing linear systems, we get

$$
\begin{aligned}
& h_{20}=\left(2 i \omega_{0} I_{n}-A\right)^{-1} B(q, q), \\
& h_{11}=-A^{-1} B(q, \bar{q}) .
\end{aligned}
$$

The coefficient in front of the $w^{3}$-term in (7.1) leads to the following expression for $h_{30}$ :

$$
h_{30}=\left(3 i \omega_{0} I_{n}-A\right)^{-1}\left[C(q, q, q)+3 B\left(q, h_{20}\right)\right]
$$

while the $w^{2} \bar{w}$-terms give the singular system for $h_{21}$ :

$$
\left(i \omega_{0} I_{n}-A\right) h_{21}=C(q, q, \bar{q})+B\left(\bar{q}, h_{20}\right)+2 B\left(q, h_{11}\right)-G_{21} q .
$$

The solvability of this system is equivalent to

$$
\left\langle p, C(q, q, \bar{q})+B\left(\bar{q}, h_{20}\right)+2 B\left(q, h_{11}\right)-G_{21} q\right\rangle=0,
$$

so the cubic normal form coefficient can be expressed as

$$
G_{21}=\left\langle p, C(q, q, \bar{q})+B\left(\bar{q},\left(2 i \omega_{0} I_{n}-A\right)^{-1} B(q, q)\right)-2 B\left(q, A^{-1} B(q, \bar{q})\right)\right\rangle
$$

and $l_{1}=\frac{1}{2} \operatorname{Re} G_{21}$ coincides with (2.5). Then,

$$
h_{21}=\left(i \omega_{0} I_{n}-A\right)^{I N V}\left[C(q, q, \bar{q})+B\left(\bar{q}, h_{20}\right)+2 B\left(q, h_{11}\right)-G_{21} q\right] .
$$

Here the complex vector $h_{21}$ satisfying $\left\langle p, h_{21}\right\rangle=0$ can be found by solving the nonsingular $(n+1)$-dimensional complex system

$$
\left(\begin{array}{cc}
i \omega_{0} I_{n}-A & q \\
\bar{p}^{T} & 0
\end{array}\right)\left(\begin{array}{c}
h_{21} \\
s
\end{array}\right)=\left(\begin{array}{c}
C(q, q, \bar{q})+B\left(\bar{q}, h_{20}\right)+2 B\left(q, h_{11}\right)-G_{21} q \\
0
\end{array}\right)
$$

For the fourth-order coefficients, we get

$$
\begin{aligned}
h_{40}= & \left(4 i \omega_{0} I_{n}-A\right)^{-1}\left[D(q, q, q, q)+6 C\left(q, q, h_{20}\right)+4 B\left(q, h_{30}\right)+3 B\left(h_{20}, h_{20}\right)\right], \\
h_{31}= & \left(2 i \omega_{0} I_{n}-A\right)^{-1}\left[D(q, q, q, \bar{q})+3 C\left(q, q, h_{11}\right)+3 C\left(q, \bar{q}, h_{20}\right)+3 B\left(h_{20}, h_{11}\right)\right. \\
& \left.\quad+B\left(\bar{q}, h_{30}\right)+3 B\left(q, h_{21}\right)-3 G_{21} h_{20}\right] \\
h_{22}= & -A^{-1}\left[D(q, q, \bar{q}, \bar{q})+4 C\left(q, \bar{q}, h_{11}\right)+C\left(\bar{q}, \bar{q}, h_{20}\right)+C\left(q, q, \bar{h}_{20}\right)\right. \\
& \left.+2 B\left(h_{11}, h_{11}\right)+2 B\left(q, \bar{h}_{21}\right)+2 B\left(\bar{q}, h_{21}\right)+B\left(\bar{h}_{20}, h_{20}\right)-2 h_{11}\left(G_{21}+\bar{G}_{21}\right)\right] .
\end{aligned}
$$

Taking into account the equality $\left\langle p, h_{21}\right\rangle=0$, one can check that the solvability condition of the linear system for $h_{32}$ provides the following formula for $l_{2}$ in (3.3):

$$
l_{2}=\frac{1}{12} \operatorname{Re} G_{32}
$$


where

$$
\begin{aligned}
G_{32}= & \langle p, E(q, q, q, \bar{q}, \bar{q}) \\
& +D\left(q, q, q, \bar{h}_{20}\right)+3 D\left(q, \bar{q}, \bar{q}, h_{20}\right)+6 D\left(q, q, \bar{q}, h_{11}\right) \\
& +C\left(\bar{q}, \bar{q}, h_{30}\right)+3 C\left(q, q, \bar{h}_{21}\right)+6 C\left(q, \bar{q}, h_{21}\right)+3 C\left(q, \bar{h}_{20}, h_{20}\right) \\
& +6 C\left(q, h_{11}, h_{11}\right)+6 C\left(\bar{q}, h_{20}, h_{11}\right) \\
& \left.+2 B\left(\bar{q}, h_{31}\right)+3 B\left(q, h_{22}\right)+B\left(\bar{h}_{20}, h_{30}\right)+3 B\left(\bar{h}_{21}, h_{20}\right)+6 B\left(h_{11}, h_{21}\right)\right\rangle
\end{aligned}
$$

with all $h_{j k}$ defined earlier. Notice that $h_{40}$ does not enter the expression for $G_{32}$. Also recall that at the Bautin bifurcation $l_{1}=0$ or $G_{21}+\bar{G}_{21}=0$, so the last term in $h_{22}$ vanishes.

8. Fold-Hopf bifurcation. At this bifurcation the system (1.1) has an equilibrium with a simple zero eigenvalue $\lambda_{1}=0$ and a pair of purely imaginary simple eigenvalues of the Jacobian matrix $A=f_{x}(0,0)$ :

$$
\lambda_{1}=0, \quad \lambda_{2,3}= \pm i \omega_{0},
$$

with $\omega_{0}>0$, and no other critical eigenvalues. Introduce two eigenvectors, $q_{0} \in \mathbf{R}^{n}$ and $q_{1} \in \mathbf{C}^{n}$,

$$
A q_{0}=0, \quad A q_{1}=i \omega_{0} q_{1}
$$

and two adjoint eigenvectors, $p_{0} \in \mathbf{R}^{n}$ and $p_{1} \in \mathbf{C}^{n}$,

$$
A^{T} p_{0}=0, \quad A^{T} p_{1}=-i \omega_{0} p_{1} .
$$

Normalize them such that

$$
\left\langle p_{0}, q_{0}\right\rangle=\left\langle p_{1}, q_{1}\right\rangle=1 .
$$

The following orthogonality properties hold: $\left\langle p_{1}, q_{0}\right\rangle=\left\langle p_{0}, q_{1}\right\rangle=0$. Now any vector $y \in T^{c} \in \mathbf{R}^{n}$ can be represented as $y=w_{0} q_{0}+w_{1} q_{1}+\bar{w}_{1} \bar{q}_{1}$, where $w_{0}=\left\langle p_{0}, y\right\rangle \in \mathbf{R}^{1}$ and $w_{1}=\left\langle p_{1}, y\right\rangle \in \mathbf{C}^{1}$. The homological equation (4.4) can be written as

$$
H_{w_{0}} \dot{w}_{0}+H_{w_{1}} \dot{w}_{1}+H_{\bar{w}_{1}} \dot{\bar{w}}_{1}=F\left(H\left(w_{0}, w_{1}, \bar{w}_{1}\right)\right)
$$

where

$$
\begin{aligned}
F(H)= & A H+\frac{1}{2} B(H, H)+\frac{1}{6} C(H, H, H)+O\left(\|H\|^{4}\right), \\
H\left(w_{0}, w_{1}, \bar{w}_{1}\right)= & w_{0} q_{0}+w_{1} q_{1}+\bar{w}_{1} \bar{q}_{1} \\
& +\sum_{2 \leq j+k+l \leq 3} \frac{1}{j ! k ! l !} h_{j k l} w_{0}^{j} w_{1}^{k} \bar{w}_{1}^{l}+O\left(\left\|\left(w_{0}, w_{1}, \bar{w}_{1}\right)\right\|^{4}\right),
\end{aligned}
$$

$h_{j k l} \in \mathbf{C}^{n}, h_{j l k}=\bar{h}_{j k l}$, and $\left(\dot{w}_{0}, \dot{w}_{1}\right)$ are defined by (3.4).

Collecting the $w_{0}^{j} w_{1}^{k} \bar{w}_{1}^{l}$-terms in (8.1) with $j+k+l=2$, one gets from the solvability conditions the expressions for the quadratic coefficients in (3.4):

$$
G_{200}=\left\langle p_{0}, B\left(q_{0}, q_{0}\right)\right\rangle, \quad G_{110}=\left\langle p_{1}, B\left(q_{0}, q_{1}\right)\right\rangle, \quad G_{011}=\left\langle p_{0}, B\left(q_{1}, \bar{q}_{1}\right)\right\rangle,
$$


and the following formulas for the coefficients $h_{j k l}$ with $j+k+l=2$ :

$$
\begin{aligned}
& h_{200}=-A^{I N V}\left[B\left(q_{0}, q_{0}\right)-\left\langle p_{0}, B\left(q_{0}, q_{0}\right)\right\rangle q_{0}\right], \\
& h_{020}=\left(2 i \omega_{0} I_{n}-A\right)^{-1} B\left(q_{1}, q_{1}\right), \\
& h_{110}=\left(i \omega_{0} I_{n}-A\right)^{I N V}\left[B\left(q_{0}, q_{1}\right)-\left\langle p_{1}, B\left(q_{0}, q_{1}\right)\right\rangle q_{1}\right], \\
& h_{011}=-A^{I N V}\left[B\left(q_{1}, \bar{q}_{1}\right)-\left\langle p_{0}, B\left(q_{1}, \bar{q}_{1}\right)\right\rangle q_{0}\right] .
\end{aligned}
$$

Here the vectors $h_{200}$ and $h_{011}$ can be computed by solving the nonsingular $(n+1)$ dimensional real systems

$$
\left(\begin{array}{cc}
A & q_{0} \\
p_{0}^{T} & 0
\end{array}\right)\left(\begin{array}{c}
h_{200} \\
s
\end{array}\right)=\left(\begin{array}{c}
-B\left(q_{0}, q_{0}\right)+\left\langle p_{0}, B\left(q_{0}, q_{0}\right)\right\rangle q_{0} \\
0
\end{array}\right)
$$

and

$$
\left(\begin{array}{cc}
A & q_{0} \\
p_{0}^{T} & 0
\end{array}\right)\left(\begin{array}{c}
h_{011} \\
s
\end{array}\right)=\left(\begin{array}{c}
-B\left(q_{1}, \bar{q}_{1}\right)+\left\langle p_{0}, B\left(q_{1}, \bar{q}_{1}\right)\right\rangle q_{0} \\
0
\end{array}\right)
$$

while the vector $h_{110}$ can be found by solving the nonsingular $(n+1)$-dimensional complex system

$$
\left(\begin{array}{cc}
i \omega_{0} I_{n}-A & q_{1} \\
\bar{p}_{1}^{T} & 0
\end{array}\right)\left(\begin{array}{c}
h_{110} \\
s
\end{array}\right)=\left(\begin{array}{c}
B\left(q_{0}, q_{1}\right)-\left\langle p_{1}, B\left(q_{0}, q_{1}\right)\right\rangle q_{1} \\
0
\end{array}\right) .
$$

Finally, the solvability conditions applied to the systems resulting from the resonant $w_{0}^{j} w_{1}^{k} \widetilde{w}_{1}^{l}$-terms in (8.1) with $j+k+l=3$ yield

$$
\begin{aligned}
& G_{300}=\left\langle p_{0}, C\left(q_{0}, q_{0}, q_{0}\right)+3 B\left(q_{0}, h_{200}\right)\right\rangle, \\
& G_{111}=\left\langle p_{0}, C\left(q_{0}, q_{1}, \bar{q}_{1}\right)+B\left(q_{0}, h_{011}\right)+B\left(q_{1}, \bar{h}_{110}\right)+B\left(\bar{q}_{1}, h_{110}\right)\right\rangle, \\
& G_{210}=\left\langle p_{1}, C\left(q_{0}, q_{0}, q_{1}\right)+2 B\left(q_{0}, h_{110}\right)+B\left(q_{1}, h_{200}\right)\right\rangle, \\
& G_{021}=\left\langle p_{1}, C\left(q_{1}, q_{1}, \bar{q}_{1}\right)+2 B\left(q_{1}, h_{011}\right)+B\left(\bar{q}_{1}, h_{020}\right)\right\rangle,
\end{aligned}
$$

where the coefficients $h_{j k l}$ are defined by (8.3)-(8.6).

9. Double Hopf bifurcation. At this bifurcation, the system (1.1) has an equilibrium with two pairs of purely imaginary simple eigenvalues of the Jacobian matrix $A=f_{x}(0,0)$ :

$$
\lambda_{1,4}= \pm i \omega_{1}, \quad \lambda_{2,3}= \pm i \omega_{2},
$$

with $\omega_{1}>\omega_{2}>0$, and no other critical eigenvalues. Assume that the conditions (3.5) hold. Since the eigenvalues are simple, there are two complex eigenvectors, $q_{1,2} \in \mathbf{C}^{n}$, corresponding to these eigenvalues:

$$
A q_{1}=i \omega_{1} q_{1}, \quad A q_{2}=i \omega_{2} q_{2} .
$$

Introduce the adjoint eigenvectors $p_{1,2} \in \mathbf{C}^{n}$ by

$$
A^{T} p_{1}=-i \omega_{1} p_{1}, \quad A^{T} p_{2}=-i \omega_{2} p_{2},
$$

where $T$ denotes transposition. These eigenvectors can be normalized using the standard scalar product in $\mathbf{C}^{n}$, 


$$
\left\langle p_{1}, q_{1}\right\rangle=\left\langle p_{2}, q_{2}\right\rangle=1
$$

and satisfy the orthogonality conditions $\left\langle p_{2}, q_{1}\right\rangle=\left\langle p_{1}, q_{2}\right\rangle=0$. Any vector $y \in T^{c} \subset$ $\mathbf{R}^{n}$ from the critical eigenspace can be represented as

$$
y=w_{1} q_{1}+\bar{w}_{1} \bar{q}_{1}+w_{2} q_{2}+\bar{w}_{2} \bar{q}_{2}, \quad w_{i} \in \mathbf{C}^{1},
$$

where $w_{1}=\left\langle p_{1}, y\right\rangle, w_{2}=\left\langle p_{2}, y\right\rangle$. Therefore, the homological equation (4.4) can be written as

$$
H_{w_{1}} \dot{w}_{1}+H_{\bar{w}_{1}} \dot{\bar{w}}_{1}+H_{w_{2}} \dot{w}_{2}+H_{\bar{w}_{2}} \dot{\bar{w}}_{2}=F\left(H\left(w_{1}, \bar{w}_{1}, w_{2}, \bar{w}_{2}\right)\right)
$$

where $F$ is defined by (2.1),

$H\left(w_{1}, \bar{w}_{1}, w_{2}, \bar{w}_{2}\right)=w_{1} q_{1}+\bar{w}_{1} \bar{q}_{1}+w_{2} q_{2}+\bar{w}_{2} \bar{q}_{2}+\sum_{j+k+l+m \geq 2} \frac{1}{j ! k ! l ! m !} h_{j k l m} w_{1}^{j} \bar{w}_{1}^{k} w_{2}^{l} \bar{w}_{2}^{m}$,

$h_{j k l m} \in \mathbf{C}^{n}, h_{k j m l}=\bar{h}_{j k l m}$, and $\left(\dot{w}_{1}, \dot{\bar{w}}_{2}\right)$ are specified by the normal form (3.6).

Collecting the coefficients of the $w_{1}^{j} \bar{w}_{1}^{k} w_{2}^{l} \bar{w}_{2}^{m}$-terms with $j+k+l+m=2$ in (9.1) gives the following expressions for $h_{j k l m}$ :

$$
\begin{aligned}
h_{1100} & =-A^{-1} B\left(q_{1}, \bar{q}_{1}\right), \\
h_{2000} & =\left(2 i \omega_{1} I_{n}-A\right)^{-1} B\left(q_{1}, q_{1}\right), \\
h_{1010} & =\left[i\left(\omega_{1}+\omega_{2}\right) I_{n}-A\right]^{-1} B\left(q_{1}, q_{2}\right), \\
h_{1001} & =\left[i\left(\omega_{1}-\omega_{2}\right) I_{n}-A\right]^{-1} B\left(q_{1}, \bar{q}_{2}\right), \\
h_{0020} & =\left(2 i \omega_{2} I_{n}-A\right)^{-1} B\left(q_{2}, q_{2}\right), \\
h_{0011} & =-A^{-1} B\left(q_{2}, \bar{q}_{2}\right) .
\end{aligned}
$$

All matrices involved in (9.3)-(9.7) are invertible in the ordinary sense due to the assumptions (3.5) on the critical eigenvalues.

Collecting the coefficients in front of the nonresonant $w_{1}^{j} \bar{w}_{1}^{k} w_{2}^{l} \bar{w}_{2}^{m}$-terms with $j+k+l+m=3$ in (9.1), one obtains the following expressions for $h_{j k l m}$ :

(9.9) $h_{2010}=\left[i\left(2 \omega_{1}+\omega_{2}\right) I_{n}-A\right]^{-1}\left[C\left(q_{1}, q_{1}, q_{2}\right)+B\left(h_{2000}, q_{2}\right)+2 B\left(h_{1010}, q_{1}\right)\right]$,

(9.10) $h_{2001}=\left[i\left(2 \omega_{1}-\omega_{2}\right) I_{n}-A\right]^{-1}\left[C\left(q_{1}, q_{1}, \bar{q}_{2}\right)+B\left(h_{2000}, \bar{q}_{2}\right)+2 B\left(h_{1001}, q_{1}\right)\right]$,

(9.11) $h_{1020}=\left[i\left(\omega_{1}+2 \omega_{2}\right) I_{n}-A\right]^{-1}\left[C\left(q_{1}, q_{2}, q_{2}\right)+B\left(h_{0020}, q_{1}\right)+2 B\left(h_{1010}, q_{2}\right)\right]$,

(9.12) $h_{1002}=\left[i\left(\omega_{1}-2 \omega_{2}\right) I_{n}-A\right]^{-1}\left[C\left(q_{1}, \bar{q}_{2}, \bar{q}_{2}\right)+B\left(\bar{h}_{0020}, q_{1}\right)+2 B\left(h_{1001}, \bar{q}_{2}\right)\right]$,

(9.13) $h_{0030}=\left(3 i \omega_{2} I_{n}-A\right)^{-1}\left[C\left(q_{2}, q_{2}, q_{2}\right)+3 B\left(h_{0020}, q_{2}\right)\right]$.

The matrices in (9.8)-(9.13) are invertible. Collecting the coefficients of the resonant cubic terms in (9.1), one obtains the resonant cubic coefficients in the normal form

$$
\begin{aligned}
& G_{2100}=\left\langle p_{1}, C\left(q_{1}, q_{1}, \bar{q}_{1}\right)+B\left(h_{2000}, \bar{q}_{1}\right)+2 B\left(h_{1100}, q_{1}\right)\right\rangle, \\
& G_{1011}=\left\langle p_{1}, C\left(q_{1}, q_{2}, \bar{q}_{2}\right)+B\left(h_{1010}, \bar{q}_{2}\right)+B\left(h_{1001}, q_{2}\right)+B\left(h_{0011}, q_{1}\right)\right\rangle, \\
& G_{1110}=\left\langle p_{2}, C\left(q_{1}, \bar{q}_{1}, q_{2}\right)+B\left(h_{1100}, q_{2}\right)+B\left(h_{1010}, \bar{q}_{1}\right)+B\left(\bar{h}_{1001}, q_{1}\right)\right\rangle, \\
& G_{0021}=\left\langle p_{2}, C\left(q_{2}, q_{2}, \bar{q}_{2}\right)+B\left(h_{0020}, \bar{q}_{2}\right)+2 B\left(h_{0011}, q_{2}\right)\right\rangle,
\end{aligned}
$$


and the corresponding cubic coefficients $h_{j k l m}$ satisfying the orthogonality conditions:

$$
\begin{aligned}
h_{2100}= & \left(i \omega_{1} I_{n}-A\right)^{I N V}\left[C\left(q_{1}, q_{1}, \bar{q}_{1}\right)+B\left(h_{2000}, \bar{q}_{1}\right)+2 B\left(h_{1100}, q_{1}\right)-G_{2100} q_{1}\right], \\
h_{1011}= & \left(i \omega_{1} I_{n}-A\right)^{I N V}\left[C\left(q_{1}, q_{2}, \bar{q}_{2}\right)+B\left(h_{1010}, \bar{q}_{2}\right)+B\left(h_{1001}, q_{2}\right)+B\left(h_{0011}, q_{1}\right)\right. \\
& \left.-G_{1011} q_{1}\right], \\
h_{1110}= & \left(i \omega_{2} I_{n}-A\right)^{I N V}\left[C\left(q_{1}, \bar{q}_{1}, q_{2}\right)+B\left(h_{1100}, q_{2}\right)+B\left(h_{1010}, \bar{q}_{1}\right)+B\left(\bar{h}_{1001}, q_{1}\right)\right. \\
& \left.-G_{1110} q_{2}\right] \\
h_{0021}= & \left(i \omega_{2} I_{n}-A\right)^{I N V}\left[C\left(q_{2}, q_{2}, \bar{q}_{2}\right)+B\left(h_{0020}, \bar{q}_{2}\right)+2 B\left(h_{0011}, q_{2}\right)-G_{0021} q_{2}\right] .
\end{aligned}
$$

Here the vectors of the form $h=\left(i \omega_{i} I_{n}-A\right)^{I N V} b$ can be found by solving the nonsingular $(n+1)$-dimensional complex systems

$$
\left(\begin{array}{cc}
i \omega_{i} I_{n}-A & q_{i} \\
\bar{p}_{i}^{T} & 0
\end{array}\right)\left(\begin{array}{c}
h \\
s
\end{array}\right)=\left(\begin{array}{l}
b \\
0
\end{array}\right) .
$$

Collecting the coefficients of the $w_{1}^{j} \bar{w}_{1}^{k} w_{2}^{l} \bar{w}_{2}^{m}$-terms with $j+k+l+m=4$ in (9.1) gives the following expressions for $h_{j k l m}$ :

$$
\begin{aligned}
& h_{4000}=\left(4 i \omega_{1} I_{n}-A\right)^{-1}\left[3 B\left(h_{2000}, h_{2000}\right)+4 B\left(h_{3000}, q_{1}\right)+6 C\left(h_{2000}, q_{1}, q_{1}\right)\right. \\
& \left.+D\left(q_{1}, q_{1}, q_{1}, q_{1}\right)\right] \\
& h_{3100}=\left(2 i \omega_{1} I_{n}-A\right)^{-1}\left[3 B\left(h_{2000}, h_{1100}\right)+3 B\left(h_{2100}, q_{1}\right)+3 C\left(h_{1100}, q_{1}, q_{1}\right)\right. \\
& \left.+3 C\left(h_{2000}, q_{1}, \bar{q}_{1}\right)+D\left(q_{1}, q_{1}, q_{1}, \bar{q}_{1}\right)-3 G_{2100} h_{2000}\right], \\
& h_{3010}=\left[i\left(3 \omega_{1}+\omega_{2}\right) I_{n}-A\right]^{-1}\left[3 B\left(h_{2010}, q_{1}\right)+3 B\left(h_{2000}, h_{1010}\right)+B\left(h_{3000}, q_{2}\right)\right. \\
& \left.+3 C\left(h_{2000}, q_{1}, q_{2}\right)+3 C\left(h_{1010}, q_{1}, q_{1}\right)+D\left(q_{1}, q_{1}, q_{1}, q_{2}\right)\right] \text {, } \\
& h_{3001}=\left[i\left(3 \omega_{1}-\omega_{2}\right) I_{n}-A\right]^{-1}\left[3 B\left(h_{2000}, h_{1001}\right)+3 B\left(h_{2001}, q_{1}\right)+B\left(h_{3000}, \bar{q}_{2}\right)\right. \\
& \left.+3 C\left(h_{2000}, q_{1}, \bar{q}_{2}\right)+3 C\left(h_{1001}, q_{1}, q_{1}\right)+D\left(q_{1}, q_{1}, q_{1}, \bar{q}_{2}\right)\right] \text {, } \\
& h_{2200}=-A^{-1}\left[2 B\left(h_{1100}, h_{1100}\right)+B\left(h_{2000}, \bar{h}_{2000}\right)+2 B\left(\bar{h}_{2100}, q_{1}\right)+2 B\left(h_{2100}, \bar{q}_{1}\right)\right. \\
& +C\left(\bar{h}_{2000}, q_{1}, q_{1}\right)+4 C\left(h_{1100}, q_{1}, \bar{q}_{1}\right)+C\left(h_{2000}, \bar{q}_{1}, \bar{q}_{1}\right) \\
& \left.+D\left(q_{1}, q_{1}, \bar{q}_{1}, \bar{q}_{1}\right)-4 \operatorname{Re}\left(G_{2100}\right) h_{1100}\right] \\
& h_{2110}=\left[i\left(\omega_{1}+\omega_{2}\right) I_{n}-A\right]^{-1}\left[2 B\left(h_{1100}, h_{1010}\right)+B\left(h_{2010}, \bar{q}_{1}\right)+2 B\left(h_{1110}, q_{1}\right)\right. \\
& +B\left(h_{2000}, \bar{h}_{1001}\right)+B\left(h_{2100}, q_{2}\right)+C\left(h_{2000}, \bar{q}_{1}, q_{2}\right) \\
& +2 C\left(h_{1100}, q_{1}, q_{2}\right)+C\left(\bar{h}_{1001}, q_{1}, q_{1}\right)+2 C\left(h_{1010}, q_{1}, \bar{q}_{1}\right) \\
& \left.+D\left(q_{1}, q_{1}, \bar{q}_{1}, q_{2}\right)-\left(G_{2100}+2 G_{1110}\right) h_{1010}\right] \\
& h_{2101}=\left[i\left(\omega_{1}-\omega_{2}\right) I_{n}-A\right]^{-1}\left[2 B\left(h_{1100}, h_{1001}\right)+2 B\left(\bar{h}_{1110}, q_{1}\right)+B\left(h_{2000}, \bar{h}_{1010}\right)\right. \\
& +B\left(h_{2100}, \bar{q}_{2}\right)+B\left(h_{2001}, \bar{q}_{1}\right)+2 C\left(h_{1100}, q_{1}, \bar{q}_{2}\right) \\
& +C\left(\bar{h}_{1010}, q_{1}, q_{1}\right)+2 C\left(h_{1001}, q_{1}, \bar{q}_{1}\right)+C\left(h_{2000}, \bar{q}_{1}, \bar{q}_{2}\right) \\
& \left.+D\left(q_{1}, q_{1}, \bar{q}_{1}, \bar{q}_{2}\right)-\left(2 \bar{G}_{1110}+G_{2100}\right) h_{1001}\right] \\
& h_{2020}=\left[2 i\left(\omega_{1}+\omega_{2}\right) I_{n}-A\right]^{-1}\left[2 B\left(h_{1020}, q_{1}\right)+2 B\left(h_{2010}, q_{2}\right)+B\left(h_{2000}, h_{0020}\right)\right. \\
& +2 B\left(h_{1010}, h_{1010}\right)+C\left(h_{2000}, q_{2}, q_{2}\right)+4 C\left(h_{1010}, q_{1}, q_{2}\right) \\
& \left.+C\left(h_{0020}, q_{1}, q_{1}\right)+D\left(q_{1}, q_{1}, q_{2}, q_{2}\right)\right] \text {, } \\
& h_{2011}=\left(2 i \omega_{1} I_{n}-A\right)^{-1}\left[B\left(h_{2010}, \bar{q}_{2}\right)+B\left(h_{2000}, h_{0011}\right)+B\left(h_{2001}, q_{2}\right)\right. \\
& +2 B\left(h_{1010}, h_{1001}\right)+2 B\left(h_{1011}, q_{1}\right)+C\left(h_{2000}, q_{2}, \bar{q}_{2}\right)
\end{aligned}
$$




$$
\begin{aligned}
& +2 C\left(h_{1010}, q_{1}, \bar{q}_{2}\right)+2 C\left(h_{1001}, q_{1}, q_{2}\right)+C\left(h_{0011}, q_{1}, q_{1}\right) \\
& \left.+D\left(q_{1}, q_{1}, q_{2}, \bar{q}_{2}\right)-2 G_{1011} h_{2000}\right] \text {, } \\
& h_{2002}=\left[2 i\left(\omega_{1}-\omega_{2}\right) I_{n}-A\right]^{-1}\left[2 B\left(h_{1002}, q_{1}\right)+B\left(h_{2000}, \bar{h}_{0020}\right)+2 B\left(h_{2001}, \bar{q}_{2}\right)\right. \\
& +2 B\left(h_{1001}, h_{1001}\right)+C\left(h_{2000}, \bar{q}_{2}, \bar{q}_{2}\right)+C\left(\bar{h}_{0020}, q_{1}, q_{1}\right) \\
& \left.+4 C\left(h_{1001}, q_{1}, \bar{q}_{2}\right)+D\left(q_{1}, q_{1}, \bar{q}_{2}, \bar{q}_{2}\right)\right] \text {, } \\
& h_{1120}=\left(2 i \omega_{2} I_{n}-A\right)^{-1}\left[B\left(h_{1100}, h_{0020}\right)+B\left(h_{1020}, \bar{q}_{1}\right)+2 B\left(h_{1110}, q_{2}\right)\right. \\
& +B\left(\bar{h}_{1002}, q_{1}\right)+2 B\left(h_{1010}, \bar{h}_{1001}\right)+C\left(h_{1100}, q_{2}, q_{2}\right) \\
& +2 C\left(\bar{h}_{1001}, q_{1}, q_{2}\right)+C\left(h_{0020}, q_{1}, \bar{q}_{1}\right)+2 C\left(h_{1010}, \bar{q}_{1}, q_{2}\right) \\
& \left.+D\left(q_{1}, \bar{q}_{1}, q_{2}, q_{2}\right)-2 G_{1110} h_{0020}\right] \text {, } \\
& h_{1111}=-A^{-1}\left[B\left(h_{1100}, h_{0011}\right)+B\left(h_{1110}, \bar{q}_{2}\right)+B\left(\bar{h}_{1110}, q_{2}\right)+B\left(\bar{h}_{1011}, q_{1}\right)\right. \\
& +B\left(\bar{h}_{1001}, h_{1001}\right)+B\left(h_{1010}, \bar{h}_{1010}\right)+B\left(h_{1011}, \bar{q}_{1}\right) \\
& +C\left(h_{1100}, q_{2}, \bar{q}_{2}\right)+C\left(\bar{h}_{1010}, q_{1}, q_{2}\right)+C\left(\bar{h}_{1001}, q_{1}, \bar{q}_{2}\right) \\
& +C\left(h_{1010}, \bar{q}_{1}, \bar{q}_{2}\right)+C\left(h_{1001}, \bar{q}_{1}, q_{2}\right)+C\left(h_{0011}, q_{1}, \bar{q}_{1}\right) \\
& \left.+D\left(q_{1}, \bar{q}_{1}, q_{2}, \bar{q}_{2}\right)-2 \operatorname{Re}\left(G_{1011}\right) h_{1100}-2 \operatorname{Re}\left(G_{1110}\right) h_{0011}\right] \text {, } \\
& h_{1030}=\left[3 i\left(\omega_{1}+\omega_{2}\right) I_{n}-A\right]^{-1}\left[3 B\left(h_{1020}, q_{2}\right)+3 B\left(h_{1010}, h_{0020}\right)+B\left(h_{0030}, q_{1}\right)\right. \\
& \left.+3 C\left(h_{0020}, q_{1}, q_{2}\right)+3 C\left(h_{1010}, q_{2}, q_{2}\right)+D\left(q_{1}, q_{2}, q_{2}, q_{2}\right)\right] \text {, } \\
& h_{1021}=\left[i\left(\omega_{1}+\omega_{2}\right) I_{n}-A\right]^{-1}\left[B\left(h_{1020}, \bar{q}_{2}\right)+B\left(h_{0020}, h_{1001}\right)+2 B\left(h_{1010}, h_{0011}\right)\right. \\
& +B\left(h_{0021}, q_{1}\right)+2 B\left(h_{1011}, q_{2}\right)+C\left(h_{1001}, q_{2}, q_{2}\right) \\
& +C\left(h_{0020}, q_{1}, \bar{q}_{2}\right)+2 C\left(h_{1010}, q_{2}, \bar{q}_{2}\right)+2 C\left(h_{0011}, q_{1}, q_{2}\right) \\
& \left.+D\left(q_{1}, q_{2}, q_{2}, \bar{q}_{2}\right)-\left(2 G_{1011}+G_{0021}\right) h_{1010}\right] \text {, } \\
& h_{1012}=\left[i\left(\omega_{1}-\omega_{2}\right) I_{n}-A\right]^{-1}\left[B\left(h_{1002}, q_{2}\right)+B\left(h_{1010}, \bar{h}_{0020}\right)+2 B\left(h_{1001}, h_{0011}\right)\right. \\
& +2 B\left(h_{1011}, \bar{q}_{2}\right)+B\left(\bar{h}_{0021}, q_{1}\right)+C\left(h_{1010}, \bar{q}_{2}, \bar{q}_{2}\right) \\
& +2 C\left(h_{1001}, q_{2}, \bar{q}_{2}\right)+C\left(\bar{h}_{0020}, q_{1}, q_{2}\right)+2 C\left(h_{0011}, q_{1}, \bar{q}_{2}\right) \\
& \left.+D\left(q_{1}, q_{2}, \bar{q}_{2}, \bar{q}_{2}\right)-\left(2 G_{1011}+\bar{G}_{0021}\right) h_{1001}\right] \text {, } \\
& h_{1003}=\left[i\left(\omega_{1}-3 \omega_{2}\right) I_{n}-A\right]^{-1}\left[3 B\left(h_{1002}, \bar{q}_{2}\right)+B\left(\bar{h}_{0030}, q_{1}\right)+3 B\left(h_{1001}, \bar{h}_{0020}\right)\right. \\
& \left.+3 C\left(\bar{h}_{0020}, q_{1}, \bar{q}_{2}\right)+3 C\left(h_{1001}, \bar{q}_{2}, \bar{q}_{2}\right)-\frac{1}{2} \bar{G}_{0032} h_{1001}\right], \\
& h_{0040}=\left(4 i \omega_{2} I_{n}-A\right)^{-1}\left[3 B\left(h_{0020}, h_{0020}\right)+4 B\left(h_{0030}, q_{2}\right)+6 C\left(h_{0020}, q_{2}, q_{2}\right)\right. \\
& \left.+D\left(q_{2}, q_{2}, q_{2}, q_{2}\right)\right] \text {, } \\
& h_{0031}=\left(2 i \omega_{2} I_{n}-A\right)^{-1}\left[3 B\left(h_{0020}, h_{0011}\right)+3 B\left(h_{0021}, q_{2}\right)+B\left(h_{0030}, \bar{q}_{2}\right)\right. \\
& +3 C\left(h_{0020}, q_{2}, \bar{q}_{2}\right)+3 C\left(h_{0011}, q_{2}, q_{2}\right)+D\left(q_{2}, q_{2}, q_{2}, \bar{q}_{2}\right) \\
& \text { - 3G } G_{0021} h_{0020} \text {, } \\
& h_{0022}=-A^{-1}\left[B\left(h_{0020}, \bar{h}_{0020}\right)+2 B\left(h_{0011}, h_{0011}\right)+2 B\left(h_{0021}, \bar{q}_{2}\right)+2 B\left(\bar{h}_{0021}, q_{2}\right)\right. \\
& +C\left(h_{0020}, \bar{q}_{2}, \bar{q}_{2}\right)+4 C\left(h_{0011}, q_{2}, \bar{q}_{2}\right)+C\left(\bar{h}_{0020}, q_{2}, q_{2}\right) \\
& \left.+D\left(q_{2}, q_{2}, \bar{q}_{2}, \bar{q}_{2}\right)-4 \operatorname{Re}\left(G_{0021}\right) h_{0011}\right] \text {. }
\end{aligned}
$$

Finally, the solvability conditions applied to the systems coming out from the resonant $w_{1}^{j} \bar{w}_{1}^{k} w_{2}^{l} \bar{w}_{2}^{m}$-terms in (8.1) with $j+k+l+m=5$ yield

$$
\begin{aligned}
G_{3200}= & \left\langle p_{1}, 3 B\left(h_{2200}, q_{1}\right)+B\left(h_{3000}, \bar{h}_{2000}\right)+2 B\left(h_{3100}, \bar{q}_{1}\right)+6 B\left(h_{1100}, h_{2100}\right)\right. \\
& +3 B\left(h_{2000}, \bar{h}_{2100}\right)+6 C\left(h_{2100}, q_{1}, \bar{q}_{1}\right)+3 C\left(\bar{h}_{2100}, q_{1}, q_{1}\right)+6 C\left(h_{1100}, h_{1100}, q_{1}\right) \\
& +C\left(h_{3000}, \bar{q}_{1}, \bar{q}_{1}\right)+3 C\left(h_{2000}, \bar{h}_{2000}, q_{1}\right)+6 C\left(h_{2000}, h_{1100}, \bar{q}_{1}\right)
\end{aligned}
$$




$$
\begin{aligned}
& +6 D\left(h_{1100}, q_{1}, q_{1}, \bar{q}_{1}\right)+3 D\left(h_{2000}, q_{1}, \bar{q}_{1}, \bar{q}_{1}\right)+D\left(\bar{h}_{2000}, q_{1}, q_{1}, q_{1}\right) \\
& \left.+E\left(q_{1}, q_{1}, q_{1}, \bar{q}_{1}, \bar{q}_{1}\right)\right\rangle \text {, } \\
& G_{2111}=\left\langle p_{1}, 2 B\left(h_{1100}, h_{1011}\right)+B\left(h_{2000}, \bar{h}_{1011}\right)+B\left(\bar{h}_{1001}, h_{2001}\right)+B\left(h_{2100}, h_{0011}\right)\right. \\
& +B\left(h_{2110}, \bar{q}_{2}\right)+2 B\left(h_{1110}, h_{1001}\right)+B\left(h_{2101}, q_{2}\right)+2 B\left(h_{1111}, q_{1}\right) \\
& +2 B\left(h_{1010}, h_{1101}\right)+B\left(h_{2010}, \bar{h}_{1010}\right)+B\left(h_{2011}, \bar{q}_{1}\right)+2 C\left(h_{1100}, h_{1010}, \bar{q}_{2}\right) \\
& +C\left(h_{2000}, \bar{h}_{1010}, q_{2}\right)+C\left(h_{2000}, h_{0011}, \bar{q}_{1}\right)+C\left(h_{2000}, \bar{h}_{1001}, \bar{q}_{2}\right) \\
& +2 C\left(h_{1110}, q_{1}, \bar{q}_{2}\right)+2 C\left(h_{1100}, h_{1001}, q_{2}\right)+C\left(h_{2100}, q_{2}, \bar{q}_{2}\right) \\
& +2 C\left(h_{1100}, h_{0011}, q_{1}\right)+2 C\left(h_{1010}, \bar{h}_{1010}, q_{1}\right)+2 C\left(h_{1010}, h_{1001}, \bar{q}_{1}\right) \\
& +2 C\left(\bar{h}_{1001}, h_{1001}, q_{1}\right)+C\left(h_{2010}, \bar{q}_{1}, \bar{q}_{2}\right)+C\left(\bar{h}_{1011}, q_{1}, q_{1}\right) \\
& +2 C\left(h_{1011}, q_{1}, \bar{q}_{1}\right)+C\left(h_{2001}, \bar{q}_{1}, q_{2}\right)+2 C\left(h_{1101}, q_{1}, q_{2}\right) \\
& +D\left(h_{0011}, q_{1}, q_{1}, \bar{q}_{1}\right)+D\left(h_{2000}, \bar{q}_{1}, q_{2}, \bar{q}_{2}\right)+2 D\left(h_{1001}, q_{1}, \bar{q}_{1}, q_{2}\right) \\
& +2 D\left(h_{1010}, q_{1}, \bar{q}_{1}, \bar{q}_{2}\right)+2 D\left(h_{1100}, q_{1}, q_{2}, \bar{q}_{2}\right)+D\left(\bar{h}_{1001}, q_{1}, q_{1}, \bar{q}_{2}\right) \\
& \left.+D\left(\bar{h}_{1010}, q_{1}, q_{1}, q_{2}\right)+E\left(q_{1}, q_{1}, \bar{q}_{1}, q_{2}, \bar{q}_{2}\right)\right\rangle \text {, } \\
& G_{1022}=\left\langle p_{1}, B\left(h_{0020}, h_{1002}\right)+4 B\left(h_{0011}, h_{1011}\right)+2 B\left(h_{1010}, \bar{h}_{0021}\right)+2 B\left(h_{1012}, q_{2}\right)\right. \\
& +B\left(h_{1020}, \bar{h}_{0020}\right)+2 B\left(h_{1001}, h_{0021}\right)+2 B\left(h_{1021}, \bar{q}_{2}\right)+B\left(h_{0022}, q_{1}\right) \\
& +4 C\left(h_{1010}, h_{0011}, \bar{q}_{2}\right)+2 C\left(h_{0011}, h_{0011}, q_{1}\right)+2 C\left(h_{1010}, \bar{h}_{0020}, q_{2}\right) \\
& +4 C\left(h_{1001}, h_{0011}, q_{2}\right)+2 C\left(h_{0021}, q_{1}, \bar{q}_{2}\right)+2 C\left(h_{0020}, h_{1001}, \bar{q}_{2}\right) \\
& +C\left(h_{0020}, \bar{h}_{0020}, q_{1}\right)+2 C\left(\bar{h}_{0021}, q_{1}, q_{2}\right)+4 C\left(h_{1011}, q_{2}, \bar{q}_{2}\right) \\
& +C\left(h_{1020}, \bar{q}_{2}, \bar{q}_{2}\right)+C\left(h_{1002}, q_{2}, q_{2}\right)+4 D\left(h_{0011}, q_{1}, q_{2}, \bar{q}_{2}\right) \\
& +D\left(h_{0020}, q_{1}, \bar{q}_{2}, \bar{q}_{2}\right)+2 D\left(h_{1010}, q_{2}, \bar{q}_{2}, \bar{q}_{2}\right)+D\left(\bar{h}_{0020}, q_{1}, q_{2}, q_{2}\right) \\
& \left.+2 D\left(h_{1001}, q_{2}, q_{2}, \bar{q}_{2}\right)+E\left(q_{1}, q_{2}, q_{2}, \bar{q}_{2}, \bar{q}_{2}\right)\right\rangle \text {, } \\
& G_{2210}=\left\langle p_{2}, 4 B\left(h_{1100}, h_{1110}\right)+2 B\left(\bar{h}_{2100}, h_{1010}\right)+B\left(\bar{h}_{2000}, h_{2010}\right)+B\left(h_{2000}, \bar{h}_{2001}\right)\right. \\
& +2 B\left(h_{2100}, h_{0110}\right)+B\left(h_{2200}, q_{2}\right)+2 B\left(h_{2110}, \bar{q}_{1}\right)+2 B\left(\bar{h}_{2101}, q_{1}\right) \\
& +2 C\left(h_{2000}, \bar{h}_{1001}, \bar{q}_{1}\right)+4 C\left(h_{1100}, h_{1010}, \bar{q}_{1}\right)+2 C\left(\bar{h}_{2000}, h_{1010}, q_{1}\right) \\
& +2 C\left(h_{2100}, \bar{q}_{1}, q_{2}\right)+2 C\left(\bar{h}_{2100}, q_{1}, q_{2}\right)+4 C\left(h_{1100}, \bar{h}_{1001}, q_{1}\right) \\
& +C\left(h_{2010}, \bar{q}_{1}, \bar{q}_{1}\right)+2 C\left(h_{1100}, h_{1100}, q_{2}\right)+4 C\left(h_{1110}, q_{1}, \bar{q}_{1}\right) \\
& +C\left(\bar{h}_{2001}, q_{1}, q_{1}\right)+C\left(h_{2000}, \bar{h}_{2000}, q_{2}\right)+4 D\left(h_{1100}, q_{1}, \bar{q}_{1}, q_{2}\right) \\
& +2 D\left(\bar{h}_{1001}, q_{1}, q_{1}, \bar{q}_{1}\right)+2 D\left(h_{1010}, q_{1}, \bar{q}_{1}, \bar{q}_{1}\right)+D\left(\bar{h}_{2000}, q_{1}, q_{1}, q_{2}\right) \\
& \left.+D\left(h_{2000}, \bar{q}_{1}, \bar{q}_{1}, q_{2}\right)+E\left(q_{1}, q_{1}, \bar{q}_{1}, \bar{q}_{1}, q_{2}\right)\right\rangle \text {, } \\
& G_{1121}=\left\langle p_{2}, 2 B\left(h_{1110}, h_{0011}\right)+B\left(\bar{h}_{1002}, h_{1001}\right)+B\left(h_{1100}, h_{0021}\right)+B\left(h_{0020}, h_{1101}\right)\right. \\
& +2 B\left(\bar{h}_{1001}, h_{1011}\right)+2 B\left(\bar{h}_{1001}, h_{1011}\right)+B\left(h_{1020}, \bar{h}_{1010}\right)+B\left(h_{1120}, \bar{q}_{2}\right) \\
& +2 B\left(h_{1111}, q_{2}\right)+B\left(h_{1021}, \bar{q}_{1}\right)+2 B\left(h_{1010}, \bar{h}_{1011}\right)+B\left(\bar{h}_{1012}, q_{1}\right) \\
& +C\left(h_{1100}, h_{0020}, \bar{q}_{2}\right)+2 C\left(\bar{h}_{1001}, h_{0011}, q_{1}\right)+C\left(h_{0020}, h_{1001}, \bar{q}_{1}\right) \\
& +2 C\left(h_{1010}, h_{0011}, \bar{q}_{1}\right)+C\left(h_{0020}, \bar{h}_{1010}, q_{1}\right)+C\left(h_{1101}, q_{2}, q_{2}\right) \\
& +2 C\left(h_{1010}, \bar{h}_{1001}, \bar{q}_{2}\right)+2 C\left(h_{1010}, \bar{h}_{1010}, q_{2}\right)+C\left(\bar{h}_{1002}, q_{1}, \bar{q}_{2}\right) \\
& +2 C\left(h_{1100}, h_{0011}, q_{2}\right)+2 C\left(\bar{h}_{1001}, h_{1001}, q_{2}\right)+2 C\left(h_{1110}, q_{2}, \bar{q}_{2}\right) \\
& +C\left(h_{0021}, q_{1}, \bar{q}_{1}\right)+2 C\left(\bar{h}_{1011}, q_{1}, q_{2}\right)+C\left(h_{1020}, \bar{q}_{1}, \bar{q}_{2}\right) \\
& +2 C\left(h_{1011}, \bar{q}_{1}, q_{2}\right)+D\left(h_{0020}, q_{1}, \bar{q}_{1}, \bar{q}_{2}\right)+D\left(h_{1100}, q_{2}, q_{2}, \bar{q}_{2}\right) \\
& +2 D\left(h_{0011}, q_{1}, \bar{q}_{1}, q_{2}\right)+2 D\left(\bar{h}_{1001}, q_{1}, q_{2}, \bar{q}_{2}\right)+D\left(h_{1001}, \bar{q}_{1}, q_{2}, q_{2}\right) \\
& \left.+D\left(\bar{h}_{1010}, q_{1}, q_{2}, q_{2}\right)+2 D\left(h_{1010}, \bar{q}_{1}, q_{2}, \bar{q}_{2}\right)+E\left(q_{1}, \bar{q}_{1}, q_{2}, q_{2}, \bar{q}_{2}\right)\right\rangle \text {, }
\end{aligned}
$$




$$
\begin{aligned}
G_{0032}= & \left\langle p_{2}, 3 B\left(h_{0022}, q_{2}\right)+B\left(h_{0030}, \bar{h}_{0020}\right)+2 B\left(h_{0031}, \bar{q}_{2}\right)+6 B\left(h_{0011}, h_{0021}\right)\right. \\
& +3 B\left(h_{0020}, \bar{h}_{0021}\right)+6 C\left(h_{0011}, h_{0011}, q_{2}\right)+3 C\left(h_{0020}, \bar{h}_{0020}, q_{2}\right)+C\left(h_{0030}, \bar{q}_{2}, \bar{q}_{2}\right) \\
& +6 C\left(h_{0020}, h_{0011}, \bar{q}_{2}\right)+3 C\left(\bar{h}_{0021}, q_{2}, q_{2}\right)+6 C\left(h_{0021}, q_{2}, \bar{q}_{2}\right) \\
& +3 D\left(h_{0020}, q_{2}, \bar{q}_{2}, \bar{q}_{2}\right)+D\left(\bar{h}_{0020}, q_{2}, q_{2}, q_{2}\right)+6 D\left(h_{0011}, q_{2}, q_{2}, \bar{q}_{2}\right) \\
& \left.+E\left(q_{2}, q_{2}, q_{2}, \bar{q}_{2}, \bar{q}_{2}\right)\right\rangle .
\end{aligned}
$$

10. Example: "New" Lorenz model. Consider the following simplified model of atmospheric circulation [12]:

$$
\left\{\begin{array}{l}
\dot{x}=-y^{2}-z^{2}-a x+a F \\
\dot{y}=x y-b x z-y+G \\
\dot{z}=b x y+x z-z
\end{array}\right.
$$

where $(F, G)$ are parameters and $a=\frac{1}{4}, b=4$. It has been shown in [15] that at

$$
F_{0}=\frac{3907}{2320}=1.684051724 \ldots, \quad G_{0}=\frac{1297}{9280} \sqrt{145}=1.682968552 \ldots,
$$

the system (10.1) has the equilibrium

$$
\left(x_{0}, y_{0}, z_{0}\right)=\left(\frac{9}{8},-\frac{1}{1160} \sqrt{145}, \frac{9}{290} \sqrt{145}\right),
$$

exhibiting a fold-Hopf bifurcation. Indeed, the Jacobian matrix of (10.1) evaluated at the critical equilibrium,

$$
A=\left(\begin{array}{ccc}
-\frac{1}{4} & \frac{\sqrt{145}}{580} & -\frac{9 \sqrt{145}}{145} \\
-\frac{\sqrt{145}}{8} & \frac{1}{8} & -\frac{9}{2} \\
\frac{4 \sqrt{145}}{145} & \frac{9}{2} & \frac{1}{8}
\end{array}\right),
$$

has the eigenvalues

$$
\lambda_{1}=0, \quad \lambda_{2,3}= \pm i \omega_{0}, \quad \omega_{0}=\frac{1}{1160} \sqrt{27561455}=4.525776271 \ldots>0 .
$$

The following vectors in $\mathbf{C}^{3}$

$$
\begin{aligned}
q_{0}= & \left(1,-\frac{1007}{188065} \sqrt{145},-\frac{5252}{188065} \sqrt{145}\right)^{T} \\
q_{1}= & \left(\frac{2}{145} \sqrt{145}, 1,-\frac{1}{36}-\frac{i}{5220} \sqrt{27561455}\right)^{T}, \\
p_{0}= & \left(\frac{188065}{190079},-\frac{2594}{190079} \sqrt{145}, 0\right)^{T}, \\
p_{1}= & \left(\frac{1007}{380158} \sqrt{145}-\frac{i}{380158} \sqrt{145} \sqrt{27561455},\right. \\
& \left.\frac{188065}{380158}+\frac{i}{380158} \sqrt{27561455},-\frac{18 i}{190079} \sqrt{27561455}\right)^{T}
\end{aligned}
$$


satisfy

$$
A q_{0}=A^{T} p_{0}=0, \quad A q_{1}=i \omega_{0} q_{1}, \quad A^{T} p_{1}=-i \omega_{0} p_{1}
$$

with the normalization conditions

$$
\left\langle p_{0}, q_{0}\right\rangle=\left\langle p_{1}, q_{1}\right\rangle=1
$$

There are no cubic terms in (10.1), while the components of the bilinear function $B(p, q)$ are given at $b=4$ by the formulas:

$$
B_{i}(p, q)=p^{T} H^{i} q, \quad i=1,2,3
$$

with the Hessian matrices

$$
H^{1}=\left(\begin{array}{ccc}
0 & 0 & 0 \\
0 & -2 & 0 \\
0 & 0 & -2
\end{array}\right), \quad H^{2}=\left(\begin{array}{ccc}
0 & 1 & -4 \\
1 & 0 & 0 \\
-4 & 0 & 0
\end{array}\right), \quad H^{3}=\left(\begin{array}{ccc}
0 & 4 & 1 \\
4 & 0 & 0 \\
1 & 0 & 0
\end{array}\right) .
$$

Following the procedure described in section 8 , one gets the values for the normal form coefficients:

$$
\begin{aligned}
& G_{200}=-\frac{124102}{190079} \\
& G_{110}=\frac{252130}{190079}+\frac{141 i}{190079} \sqrt{145} \sqrt{190079} \\
& G_{011}=-\frac{6915604}{1710711} \\
& G_{300}=\frac{25602814720}{36130026241} \\
& G_{111}=\frac{9729482240}{325170236169}, \\
& G_{210}=-\frac{36640288960}{36130026241}+\frac{959598417280 i}{6867559257863039} \sqrt{145} \sqrt{190079} \\
& G_{021}=-\frac{4426799104}{325170236169}+\frac{51158402858528 i}{26886494494533797685} \sqrt{145} \sqrt{190079} .
\end{aligned}
$$

This gives the following numerical values for the coefficients of the reduced normal form of section 3 :

$$
b(0)=-\frac{62051}{190079}=-0.3264484767 \ldots, \quad c(0)=-\frac{6915604}{1710711}=-4.042532023 \ldots,
$$

while

$$
\theta(0) \equiv \frac{\operatorname{Red}(0)}{G_{200}}=-\frac{126065}{62051}=-2.031635268 \ldots<0
$$

and

$$
e(0)=\frac{816491531617717015648}{3876030514045053390191}=0.2106514716 \ldots>0
$$


Thus, the case $s=\operatorname{sign}(b c)=1, \theta<0$ occurs without time reversing (see [10, pp. 293-303] for the corresponding phase portraits). This proves the conjecture formulated in [15] on the basis of numerical analysis of bifurcations of equilibria, cycles, and homoclinic orbits in (10.1) but without actual computation of the normal form coefficients. Therefore, a nontrivial invariant set bifurcates from the critical equilibrium under parameter variations.

11. Discussion. The formulas above derived for the normal form coefficients allow us to verify the nondegeneracy conditions (see section 3 and [10]) for all codim 2 equilibrium bifurcations. In particular, computing the coefficients for the fold-Hopf and double Hopf bifurcations allows us to distinguish between "simple" and "difficult" cases implying chaotic motions. Section 10 provides an example of such an analysis.

The formulas are independent on the dimension $n$ of the phase space and involve only critical eigenvectors of $A$ and $A^{T}$. They are also valid when $n_{c}=n$ (the dimension of the center manifold is equal to the phase space dimension).

The formulas are easily programmable if algebraic operations with complex matrices are supported, as in MAPLE, Mathematica, or MATLAB. Finding the intermediate coefficients $h_{\nu}$ reduces via a bordering technique to solving nonsingular (complex) linear systems. If symbolic derivatives of the right-hand side of (1.1) are available, they can be used directly to evaluate the multilinear functions $B, C, D, E$ by the formulas given after (2.1). If no symbolic derivatives are given, these functions (and scalar products involving them) can be approximated numerically using only directional derivatives of the right-hand side of (1.1) (see [10] for finite-difference approximations of $B(p, q)$ and $C(q, q, p))$.

The formulas for the double Hopf bifurcation are complicated. The author tried to reduce the possibility of misprints by automatic conversion of the MAPLE output into $\mathrm{IT}_{\mathrm{E}} \mathrm{X}$ with minimal postprocessing. The MAPLE V.R4 sessions used to derive the coefficients in sections 7, 8, and 9 are available by anonymous ftp in pub/yuri/coeff2 on ftp.cwi.nl. In actual implementations of these formulas, the corresponding $\mathrm{C}$ or FORTRAN codes should be generated automatically.

Acknowledgment. The author is thankful to Dr. A. Heck (UvA) for his help in dealing with multilinear functions in MAPLE V.R4.

\section{REFERENCES}

[1] V. I. ARNold, Geometrical Methods in the Theory of Ordinary Differential Equations, Springer-Verlag, New York, Heidelberg, Berlin, 1983.

[2] W-J. BEYN AND W. KLESS, Numerical Taylor Expansions of Invariant Manifolds in Large Dynamical Systems, Preprint 6/96, Fakultät für Math., Univ. Bielefeld, Germany, 1996.

[3] P. Coullet And E. SPIEgel, Amplitude equations for systems with competing instabilities, SIAM J. Appl. Math., 43 (1983), pp. 776-821.

[4] C. Elphick, E. Tirapegui, M. Brachet, P. Coullet, And G. Iooss, A simple global characterization for normal forms of singular vector fields, Phys. D, 32 (1987), pp. 95-127.

[5] W. GovaERTS AND J. PRYCE, Mixed block elimination for linear systems with wider borders, IMA J. Numer. Anal., 13 (1993), pp. 161-180.

[6] J. GuCKenheimer And P. Holmes, Nonlinear Oscillations, Dynamical Systems and Bifurcations of Vector Fields, Springer-Verlag, New York, 1983.

[7] B. Hassard, N. Kazarinoff, And Y.-H. Wan, Theory and Applications of Hopf Bifurcation, Cambridge University Press, London, New York, New Rochelle, Melbourne, Sydney, 1981.

[8] H. KELLER, Numerical solution of bifurcation and nonlinear eigenvalue problems, in Applications of Bifurcation Theory, P. Rabinowitz, ed., Academic Press, New York, 1977, pp. 359384 . 
[9] L. G. Kurakin and V. I. Judovich, Semi-invariant form of equilibrium stability criteria in critical cases, J. Appl. Math. Mech., 50 (1986), pp. 543-546.

[10] Yu. A. KuzNetsov, Elements of Applied Bifurcation Theory, Springer-Verlag, New York, 1995.

[11] YU. A. KuZNETSOV AND V. V. LeVITIN, CONTENT: A Multiplatform Environment for Analyzing Dynamical Systems. Dynam. System Lab., Centrum voor Wiskunde en Inform. Amsterdam, available from ftp.cwi.nl/pub/CONTENT, 1997.

[12] E. LoREnZ, Irregularity: A fundamental property of the atmosphere, Tellus, 36A (1984), pp. 98110.

[13] J. Marsden and M. McCracken, Hopf Bifurcation and Its Applications, Springer-Verlag, New York, Heidelberg, Berlin, 1976.

[14] J. SANDERS, Versal normal form computation and representation theory, in Computational Algebra and Differential Equations, E. Tournier, ed., Cambrige University Press, UK, 1994, pp. 185-210.

[15] A. L. ShILNIKov, G. Nicolis, AND C. Nicolis, Bifurcation and predictability analysis of a low-order atmospheric circulation model, Internat. J. Bifur. Chaos Appl. Sci. Engrg., 5 (1995), pp. 1701-1711.

[16] S. VAN GILS, On a Formula for the Direction of Hopf Bifurcation, Rep. TW/225, Centrum voor Wiskunde en Inform., Amsterdam, 1982. 